\title{
La comunicación de la ciencia en la pandemia por COVID-19 y sus divulgadores
}

\section{The Communication of Science in the COVID-19 Pandemic and its Disseminators}

\author{
Lizy Navarro Zamora \\ https://orcid.org/0000-0001-7907-7729 \\ Universidad Autónoma de San Luis Potosí \\ lizy@uaslp.mx \\ México
}

\begin{abstract}
Resumen:
Ante el fenómeno de la pandemia del COVID-19, se realizó un estudio descriptivo aleatorio sobre la comunicación de la ciencia en la televisión digital abierta, los periódicos impresos y los cibermedios de México, además de monitorear actividades en redes sociales digitales. Los resultados principales son los siguientes: hubo crecimiento de la comunicación de la ciencia en la televisión, periódicos y cibermedios. En Internet y las redes sociales solo se desarrolló una mínima divulgación científica de real impacto, al igual que en las actividades presenciales; mientras que los museos en el país se convirtieron en los únicos en restablecer la comunicación directa, aunque al mínimo. De manera paralela, en septiembre de 2020 se aplicó una encuesta a 120 divulgadores de la ciencia de todo el país sobre su actividad como divulgadores en la pandemia COVID-19 durante ese año. Algunos de los resultados obtenidos muestran que no existe una formación de competencias educomunicativas de los divulgadores de la ciencia, ya que durante la pandemia no se han actualizado. Indicaron que son necesarias mayores competencias y que, según su punto de vista, la comunicación y la educación son los dos elementos fundamentales para la comunicación de la ciencia.
\end{abstract}

Palabras clave: comunicación de la ciencia; educación; COVID-19.

\begin{abstract}
:
Faced with the phenomenon of the COVID-19 pandemic, a random descriptive study on communication of science was carried out on open digital television, print newspapers and cybermedia in Mexico, in addition to monitoring activities in digital social networks. The main results are the following: there was a growth in communication of science on television, newspapers and cybermedia. On the Internet and social networks, only minimal scientific divulgation of real impact was developed; while the museums in the country became the only space to restore direct communication, albeit to a minimum. At the same time, in September 2020, a survey was applied to 120 science disseminators, from all over the country regarding their activity as divulgators in the COVID-19 pandemic during that year. Some of the results show that there is no training of science popularizers in educommunicative skills, because during the pandemic they have not been updated. The respondents noted that greater skills are needed and that, from their point of view, communication and education are the two fundamental elements for the divulgation of science.
\end{abstract}

Keywords: communication of science; education; COVID-19.

Recibido: 01/03/2021 | Aceptado: 06/05/2021 | Publicado: 07/07/2021

| pág. 82 - 109 
La comunicación de la ciencia en la pandemia por COVID-19 y sus divulgadores

\section{| Introducción}

La presente investigación científica se centró en la comunicación de la ciencia en torno a la pandemia COVID-19, de marzo a diciembre del 2020. Este tema tiene la esencia en la responsabilidad social $\mathrm{y}$, como lo define la Organización de las Naciones Unidas para la Educación, la Ciencia y la Cultura [UNESCO por sus siglas en inglés] (UNESCO, 2018), se enmarca en el objetivo de pasar de la Sociedad de la Información a la Sociedad del Conocimiento. En México aún no se llega a la primera: la comunicación de la ciencia no es un área emergente del presente siglo; sus inicios fueron en las ciencias exactas a partir del siglo XX.

En la actualidad todavía se presenta una discusión en la literatura académica en torno al tema tratado en este artículo, ya que no existen acuerdos absolutos sobre las diferencias entre la comunicación de la ciencia, la difusión de la ciencia, la divulgación de la ciencia y el periodismo científico, éste último ubicado como la comunicación de la ciencia difundida a través de la radio, la televisión, las revistas y los cibermedios. Según Calvo-Hernando (2003), uno de los investigadores enfocados en las reflexiones conceptuales de estos temas:

El concepto de divulgación científica es más amplio que el de periodismo científico... La divulgación nace en el momento en el que la comunicación de un hecho científico deja de estar reservada exclusivamente a los propios miembros de la comunidad investigadora o a las minorías que dominan el poder, la cultura o la economía. (p. 17).

Las diferencias entre los conceptos es un tema que se sigue reflexionando por los investigadores científicos de la comunicación:

La difusión y la divulgación de la ciencia son tareas fundamentales 
para el avance del conocimiento. Sin difusión es imposible que los hombres de ciencia conozcan las contribuciones y hallazgos de sus colegas, y sin divulgación se niega la oportunidad a la sociedad a que comprenda y se beneficie del progreso científico. Y lo más grave: se imposibilita que los niños y los jóvenes se interesen por la ciencia. (Martínez-Mendoza, 2012, p. 78).

Entonces trataremos la comunicación de la ciencia como la actividad dentro de la comunicación a través de la cual se transmiten mensajes científicos por diversos canales, con un emisor y un receptor. La esencia es un conocimiento científico que se transforma a través de la mediación de la comunicación y la educación, en contenido accesible para diversos grupos, por edades, por nivel socioeconómico, por lugares, y que permite un aprendizaje significativo. También dentro de la teoría de la comunicación se han trabajado estos temas:

Podemos claramente visualizar este concepto de divulgación científica como actividad de difusión en el modelo inicial del proceso comunicativo elaborado por Lasswell, en el que la difusión de informaciones presupone la estructura de un emisor, que envía un mensaje por un canal, a un receptor, causando determinado(s) efecto(s). El emisor sería el divulgador científico (el mismo científico o el profesional de comunicación), el mensaje sería el contenido de la ciencia a ser trabajado en un lenguaje accesible y a ser transmitido por los medios de comunicación a un público lego. (Ataide-Malcher \& Cunha-López, 2013, p. 78).

El tema de la comunicación de la ciencia se ha estudiado desde la investigación científica del área de la educación, del área de la comunicación, y de cada una de las disciplinas del conocimiento. En los años recientes se ha considerado un enfoque más complejo, integrando los elementos educomunicativos con el conocimiento científico. En México, el espacio que organiza la investigación científica y la divulgación de la ciencia es el Consejo Nacional de Ciencia y Tecnología (CONACYT), que desde el año 2019 ha incorporado en su 
marco de desarrollo el concepto de Apropiación Social de la Ciencia y la Tecnología, el cual significa hacer propio algo que resulta ajeno o extraño; se trata de encontrar una manera de relacionarlo y de incorporarlo en la propia vida.

La actual gestión de CONACYT le ha dado un gran énfasis a la comunicación de la ciencia, lo cual se demuestra en el Reglamento para los miembros del Sistema Nacional de Investigadores (SNI) del 2021, el cual estipula que sus miembros deben realizar también actividades de divulgación de la ciencia para pertenecer al sistema.

El conocimiento científico está en el centro de la comunicación de la ciencia y los dos pilares que le permiten su construcción son la comunicación y la educación. De qué se habla es lo esencial; sin embargo, es necesario dar énfasis en la forma de trasmitirlo y en un contexto pedagógico que permita convertirlo en un aprendizaje significativo. Para esto se pueden aplicar las teorías constructivistas de Lev Vygotsky y de David Ausubel:

En toda forma comunicativa hay aspectos educativos y viceversa; educación y comunicación están indisolublemente ligadas... la divulgación es una disciplina que se encarga de llevar el conocimiento científico y técnico a un público no especializado, que va desde los niños hasta las personas de edad. (PachecoMuñoz, 2003, p. 56).

Este reconocimiento de la comunicación y educación ha permitido mejores productos de divulgación científica que inciden en los diferentes grupos sociales, con diferencias socioeconómicas, en su nivel académico; en su lugar de residencia, desde las comunidades rurales hasta las grandes ciudades. Como investigadora científica social de temas como la comunicación, la educación, las Tecnologías de la Información y Comunicación (TIC) y la divulgación de la ciencia, considero que, tanto en el campo de la investigación, como en la construcción de productos de divulgación, es necesaria la formación en educación y comunicación.

En el pilar educativo, en la divulgación de la ciencia se incluye el Modelo Socio-Cultural de Lev Vygotsky el cual se fundamenta 
en tres zonas que son: a) zona de desarrollo real-conocimiento previo; b) zona de desarrollo próximoconocimiento nuevo y c) zona de desarrollo potencial-evaluación de conocimiento (lo aprendido). Este planteamiento presupone necesariamente que el proceso de desarrollo no coincide con el aprendizaje, el proceso de desarrollo sigue al de aprendizaje, que crea el área de desarrollo potencial.

Otra de las teorías educativas que fundamentan la construcción de contenidos es la del Aprendizaje Significativo de David Ausubel, quien ubicó cuatro etapas: 1) base previa, 2) interacción con el nuevo conocimiento, 3) relación con el nuevo conocimiento y 4) ponerlo en práctica. Los individuos buscan en su propio conocimiento personal, en sus habilidades adquiridas y disposiciones, los recursos disponibles para ellos. Como se define,

Aprendizaje significativo es aquél en el que ideas expresadas simbólicamente interactúan de manera sustantiva y no arbitraria con lo que el aprendiz ya sabe. Sustantiva quiere decir no literal, que no es al pie de la letra, y no arbitraria significa que la interacción no se produce con cualquier idea previa, sino con algún conocimiento específicamente relevante ya existente en la estructura cognitiva del sujeto que aprende... El aprendizaje significativo se caracteriza por la interacción entre conocimientos previos $\mathrm{y}$ conocimientos nuevos y que esa interacción es no literal y no arbitraria. En ese proceso, los nuevos conocimientos adquieren significado para el sujeto y los conocimientos previos adquieren nuevos significados o mayor estabilidad cognitiva. (Moreira, 2012, p. 25).

Otro elemento fundamental es el planteamiento de quién es y quién no es divulgador de la ciencia. "Entre los divulgadores, unos escriben ocasionalmente, lo mismo si son investigadores que escritores o periodistas, $y$ otros se dedican expresamente a la difusión de la ciencia y la tecnología”. (CalvoHernando, 2003, p. 112).

Y también dentro de la comunicación de la ciencia se integran elementos 
a partir del desarrollo de Internet en medio de la Pandemia COVID-19. Como lo afirman Corredor-Trejo y Socorro (2014) en su artículo "Impacto de las tecnologías de información y comunicación en la divulgación del conocimiento científico",

En la actualidad, las redes sociales son imprescindibles para la comunicación pública de la ciencia, especialmente en un ámbito en el que la ciencia ha tenido poca divulgación y repercusión en los medios de comunicación tradicionales prensa escrita, radio $\mathrm{y}$ televisión. Las redes sociales han democratizado la comunicación en general y la de la ciencia en particular. Esta apertura tecnológica comunicacional ha acercado a los investigadores a redes sociales como Facebook, Twitter, blogs especializados en ciencia y tecnología y el resto de redes sociales... (p. 153).

En esta investigación científica se consideró la comunicación de la ciencia como la transmisión de contenido científico a un público no experto en él, a través de los medios masivos de comunicación, de actividades mediadas por las Tecnologías de la Información y Comunicación (TIC), y de acciones presenciales.

Marco contextual de la comunicación de la ciencia en la pandemia COVID-19

En el 2021 continúa la pandemia del COVID-19 en todo el mundo: la vacunación ha llevado atrasos, ya que fue en diciembre del 2020 que se tuvo el biológico para proteger a los ciudadanos. De marzo a diciembre del 2020, en el mundo, principalmente en los países desarrollados, se incrementó el desarrollo científico para conocer este fenómeno, los posibles medicamentos y las vacunas. Oficialmente, se establece por la Organización Mundial de la Salud, el inicio de la pandemia COVID-19 a partir de diciembre del 2019. En diciembre del 2020 ya se contaba con las vacunas en circulación, por supuesto con una venta restringida y muy limitada hacia los gobiernos. La presente investigación, de enero a marzo del 2020, se centra en el objeto de estudio de la comunicación de la ciencia de todos los temas, ámbito que ha cobrado 
gran interés en las investigaciones científicas, desde el ángulo de la comunicación como desde la educación, independientemente del conocimiento científico de cada área del conocimiento.

Estamos inmersos en una sociedad cimentada en conocimientos científicos. La pandemia del COVID-19 se convirtió en un parteaguas para la humanidad: un antes y un después como en su momento fueron la imprenta, la Revolución Francesa, la Independencia de Estados Unidos, la Segunda Guerra Mundial, o el nacimiento de Internet. Algunos son eventos históricos que sucedieron y no fueron planeados; en el caso de Internet nació en los años setenta, fue más conocida a finales de los ochenta junto a otras redes y llegó a México en los noventa. La imprenta fue un invento que significó un parteaguas, pero Johanes Gutenberg no dimensionó la trascendencia de ello; como tampoco lo hicieron los protagonistas de la Revolución Francesa. En el libro Los periódicos On line (Navarro-Zamora, 2002), se da cuenta de todo el recorrido histórico de ello.
La COVID-19 es una pandemia que cobra dimensión por la posibilidad de la globalización que nos permite el siglo XXI, imposible en otros momentos de la humanidad. Hoy las naciones están preocupadas porque la rapidez en el alcance de la vacunación afectará o beneficiará a todos los países. La movilidad física que se permite hoy con la disminución en las horas de vuelo, para viajar de un continente a otro, las relaciones comerciales para la importación de los productos y la movilidad de trabajadores de empresas, son elementos que caracterizan al siglo XXI. En todos estos eventos humanos se ha presentado la urgencia de contar con más expertos en ciencia y en tecnología, altamente calificados, para responder a las demandas de la sociedad. Durante el 2021 se continúa la búsqueda de un medicamento efectivo, de vacunas para los niños y de un uso asertivo de Internet, etc.

En la literatura sobre el tema de la comunicación de la ciencia, también llamada divulgación científica, ya que no existen absolutos en la connotación de estos términos, se 
presentan actividades tradicionales y actividades emergentes. Dentro de los espacios de divulgación tradicionales se tienen las revistas, los museos, los talleres, las visitas guiadas a centros de investigación y universidades, así como la televisión digital abierta, la radio y los periódicos impresos, las conferencias en congresos, entre otros. En los espacios emergentes mediados por las TIC están YouTube, redes sociales y cibermedios.

Presentemos los museos como ejemplo de actividad de divulgación de la ciencia, de todas las áreas del conocimiento, desde las ciencias sociales hasta las ciencias exactas, en su forma más tradicional y más actual (UNESCO, 2020). Los museos siempre han estado en constante evolución; por ello, una de las clasificaciones actuales se basa en su interactividad $\mathrm{y}$ los ubica en generaciones.

En la primera generación de nula interactividad están los museos contemplativos, en los que predomina la museología del objeto. En este museo el visitante es pasivo y el objeto se encuentra fuera de su alcance, en un pedestal, en espacios cerrados. La mayoría de los museos en el país están en esta categoría, sobre todo los que no se ubican en las principales ciudades del país. Se puede mencionar que éstos presentan una comunicación de la ciencia con un modelo educativo tradicional.

En la segunda generación están aquellos que permiten cierto grado de interactividad, de manera directa o indirecta. Aquí ya existen recursos lúdicos, prevalece el modelo tradicional, aunque se dan algunos elementos constructivistas para los adultos. En la tercera generación de museos se promueve el enfoque educativo constructivista. El museo es un espacio de participación activa del visitante donde el objeto es el centro de la interactividad y prevalece la participación del usuario, desde los infantes hasta los mayores.

Los museos de mayor desarrollo, que son menos del $1 \%$ en México, se ubican en la cuarta generación, donde la museografía se construye a partir de un proyecto educativo y de comunicación con el usuario. La experimentación, la reflexión y la visión crítica son esenciales. Por supuesto, los mejores son los museos de esta generación; sin embargo, no 
todos los objetos en los cuales se basa la museografía pueden adaptarse a ellos. En la museografía los temas de educación y de comunicación son esenciales.

Otras actividades de comunicación de la ciencia muy importantes, de gran tradición y enfocadas a todos los espacios, tanto en las ciudades como en las comunidades rurales, son los talleres y las visitas guiadas. Éstas se han desarrollado en programas como la Semana Nacional de Ciencia y Tecnología, tanto en el ámbito de cada entidad federativa de la República Mexicana como a nivel nacional. En ellos se da un mayor grado de comunicación, interactividad $y$ sobre todo de aprendizaje significativo porque la actividad es presencial, cara a cara, tanto en capitales como en comunidades rurales. En los medios masivos se ubica la comunicación de la ciencia en la televisión digital abierta, la radio y los periódicos impresos, todos ellos entendidos como espacios para la transmisión de la información científica.

Tenemos como ejemplo de esto último los canales de televisión de las empresas Televisa y de Televisión
Azteca, que tienen espacios de comunicación de la ciencia, principalmente en sus noticiarios. De radio tenemos ejemplos como Radio Fórmula, la W Radio; en periódicos La Jornada, Excélsior y El Universal. En sí, en ellos existe un respaldo de la marca, por ser medios de comunicación con alta audiencia en el país y amplia circulación, la sociedad confía en aquello que se dice; es decir cuentan con la confianza de los televidentes, de los radioescuchas, de los lectores y de los usuarios. Se tiene y se confía en estos nombres de marca, es decir de prestigio para quien los consume. Esto, a partir del desarrollo histórico de los medios de comunicación en México (Navarro-Zamora, 2002).

También se tiene una amplia credibilidad en los cibermedios, que nacieron de periódicos impresos con un amplio prestigio y posibilidades distintas (Navarro-Zamora, 2018). Por otra parte, se han ubicado mediaciones emergentes para toda actividad humana, en las cuales se incluye la comunicación de la ciencia, como pueden ser las redes sociales digitales y espacios como YouTube. Con base en datos de la Asociación de Internet, en enero del 2021, se 
tiene que México alcanzó un 74\% de penetración entre la población de personas mayores a 6 años. De estos el $11 \%$ de los usuarios de Internet tiene de 6 a 11 años; el 13\% de 12 a 17 años; el $16 \%$ de 18 a 24 años; el $21 \%$ de 25 a 34 años; el $17 \%$ de 24 a 44 años; el $12 \%$ de 35 a 54 años y el 10\% de 55 o más años. De estos usuarios, el $51 \%$ son mujeres y el $49 \%$ son hombres. (Asociación de Internet MX, 2021).

Además, también existe una diferencia significativa de conectividad entre las zonas del país: en la Centro Sur de 24\%; en el Noroeste de 16\%; en el Centro Norte de 13\%; en el Este de1 2\%; en el Oeste de 11\%; en el Noreste 11\%; en la Suroeste $8 \%$ y en el Sureste 7\%. Aún con el crecimiento a nivel nacional se tienen realidades distintas entre los conectados a Internet y los desconectados.

\section{| Metodología}

Esta investigación, con un alcance descriptivo, se divide en dos partes: la investigación sobre los espacios mediáticos digitales, gratuitos en televisión y de pago en medios impresos, así como de cibermedios, lo que llamamos medios masivos de comunicación, que hacen comunicación de la ciencia, principalmente en sus noticiarios. Se realizó un análisis de contenido cuantitativo, para lo cual se seleccionó una muestra de televisión abierta, de periódicos nacionales impresos y de cibermedios. En la televisión se eligió el Canal 2 y Canal 5 de la empresa Televisa; el Canal 7 y Uno de Televisión Azteca y el Canal 11 del Instituto Politécnico Nacional. La revisión fue los días lunes, de marzo a diciembre del 2020 comparándolo con los mismos meses y días del 2019, en un horario de 7:00 am a 12:00 pm. En el caso de los canales de televisión se utilizó YouTube para obtener las transmisiones del 2019; así como de archivos personales.

De los medios impresos se revisaron La Jornada, Reforma, El Universal, también los lunes de cada semana de los meses de marzo a diciembre del 2020 y del 2019. Las comparaciones fueron entre los contenidos de los mismos medios de ambos años. Los periódicos impresos se obtuvieron de la hemeroteca personal $y$ los cibermedios a través de sus hemerotecas digitales. Se descartó 
la radio por ser inaccesibles sus archivos durante los meses de la pandemia, ubicados en la audioteca de la Universidad Nacional Autónoma de México [UNAM].

Para esta investigación, se consideró el objeto de estudio del 2019 como el $100 \%$ y de allí se procedió al comparativo del 2020 cuando existía la pandemia y todas las actividades sufrieron consecuencias, sobre todo en la cuarentena nacional y en las cuarentenas parciales que tuvo cada entidad federativa.

Una de las variables analizadas fue el incremento o disminución en contenidos de comunicación de la ciencia, sus temáticas, sus formas, su desarrollo. Otras variables fueron las formas de comunicación de la ciencia en los medios masivos organizados en noticias, entrevistas, crónicas, reportajes, columnas, artículos, editoriales, programas específicos de divulgación, cibergéneros, entre otros.

En cuanto a las formas tradicionales de comunicación de la ciencia como talleres, visitas guiadas $\mathrm{y}$ talleres presenciales, se analizaron las actividades de los organismos públicos que organizan eventos de Divulgación de la Ciencia, de las 32 entidades federativas y dependientes del gobierno de cada estado y que toman directrices de CONACYT; ésto en la página "web" de cada uno de los sitios. Por ello se tienen los resultados en torno a los museos, las visitas presenciales y los talleres presenciales. Igual que en los medios masivos, el estudio se realizó de marzo a diciembre del 2020 comparado con marzo a diciembre del 2019.

En torno a las redes sociales como Facebook, Tik Tok, Instagram y YouTube, se consideraron aquellas páginas y autores que emiten sus mensajes en México, con más de un millón de visitas en el 2019. Cada día, cada hora, cada segundo se abren cuentas, sin embargo la existencia no significa espacios visitados por muchas personas. Es decir, no se consideraron todas las páginas, publicaciones, ya que para el presente estudio no tienen relevancia. Por supuesto, una gran parte de la población publicó información sobre el tema de la pandemia, pero eso no es divulgación de la ciencia, es una comunicación 
libre a la cual tiene derecho todo ciudadano y quien además no tiene obligación de ejercer una actividad que requiere la responsabilidad de una formación de sus competencias. Ser divulgador de la ciencia significa tener una formación mínima de comunicación y educación, no sólo publicar contenido sobre ciencia.

Después de realizar el análisis de contenido cuantitativo y con el objetivo de profundizar en el tema, se trabajó con una segunda heramienta de recolección de información, que consistió en una encuesta aleatoria a las y los divulgadores. Para la selección de la muestra se enviaron mensajes a las cuentas en redes sociales y correos electrónicos de divulgadores. Para esto se contactó previamente a las personas registradas en la Sociedad Mexicana para la Divulgación de la Ciencia y la Técnica (SOMEDECYT) y a través de la red de la Asociación Mexicana de Investigadores de Comunicación (AMIC). Los resultados son a partir de la respuestas recibidas; en total se mandaron a 400 divulgadores de la ciencia y de ellos 127 respondieron, 7 se desecharon por no responder a los planteamientos.
Esto se realizó durante el mes de septiembre del 2020. Se consideró como representación mínima a un divulgador o una divulgadora por entidad federativa. El diseño de la encuesta incluyó 18 preguntas cerradas y tres abiertas. Los divulgadores a los cuales se encuestó no necesariamente son autores o productores de contenidos de comunicación de la ciencia analizados.

Esta encuesta se realizó con el objetivo de conocer el perfil de los divulgadores de la ciencia, su formación, las actividades que realizaron durante la pandemia de marzo a diciembre del 2020 y que posiblemente serán los protagonistas en el 2021, ya que los divulgadores serán los promotores de la divulgación no realizada, en el retorno a la normalidad tras la vacunación, a los desafíos de la llamada nueva normalidad.

\section{| Resultados}

El estudio se desarrolló durante la pandemia por COVID-19. El tema estuvo en la agenda de los medios de comunicación y de la sociedad, y seguramente fue una de las razones 
por las cuales se dio el incremento de la divulgación científica en televisión digital abierta, periódicos impresos y cibermedios.

Figura 1.

Espacios de comunicación de la ciencia que se incrementaron durante la pandemia por COVID-19.

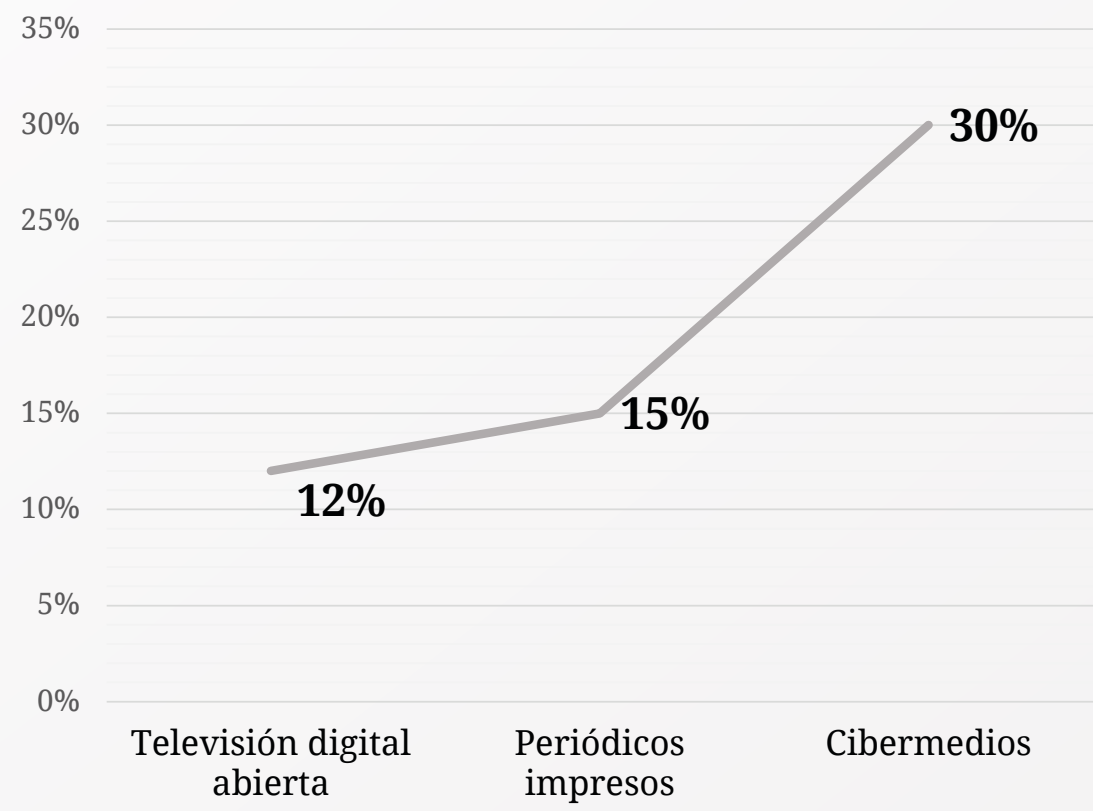

En la figura 1 se puede apreciar los periódicos como el centro de los el incremento que se tuvo en contenidos para los tomadores de la televisión digital abierta, decisiones y los cibermedios como los periódicos impresos y los los espacios de desarrollo ante los cibermedios, de marzo a diciembre del 2020 comparado con los mismos meses del 2019, este último año considerado un 100\%. Esta gráfica refleja los resultados de la Comunicación de la Ciencia en tres de los espacios de mayor acceso para el público.

De manera específica se construyen los medios de comunicación con una finalidad distinta: la televisión con el objetivo del entretenimiento, cambios de la sociedad y las TIC. Cada uno de estos espacios de contenido llega a públicos distintos (MartínezAlbertos, 1997).

Recordemos que en la metodología se especificó que se partió de las actividades del año 2019, de marzo a diciembre, las cuales significaron el $100 \%$, y se compararon con los mismos meses para el año 2020 de la pandemia por COVID-19. En la figura 2 los resultados parten del análisis 
realizado a los organismos públicos dependientes de cada una de las 32 entidades federativas y que basan sus criterios en aquellos emitidos por CONACYT.

En la figura 2 se presentan tres espacios como los museos, las visitas presenciales y los talleres. La pandemia por COVID-19 provocó que dichas actividades, hasta el mes de agosto del 2020, fueran anuladas, casi en su totalidad. Algunos de los museos, sobre todos los que pertenecen a municipios, aunque son los menos, permanecieron abiertos los meses de marzo y abril. Esto se debe a que el fenómeno de la pandemia ha tenido presencia distinta entre las localidades $\mathrm{y}$ estados del país.

\section{Figura 2.}

Espacios de Comunicación de la ciencia que disminuyeron durante la pandemia por COVID-19.

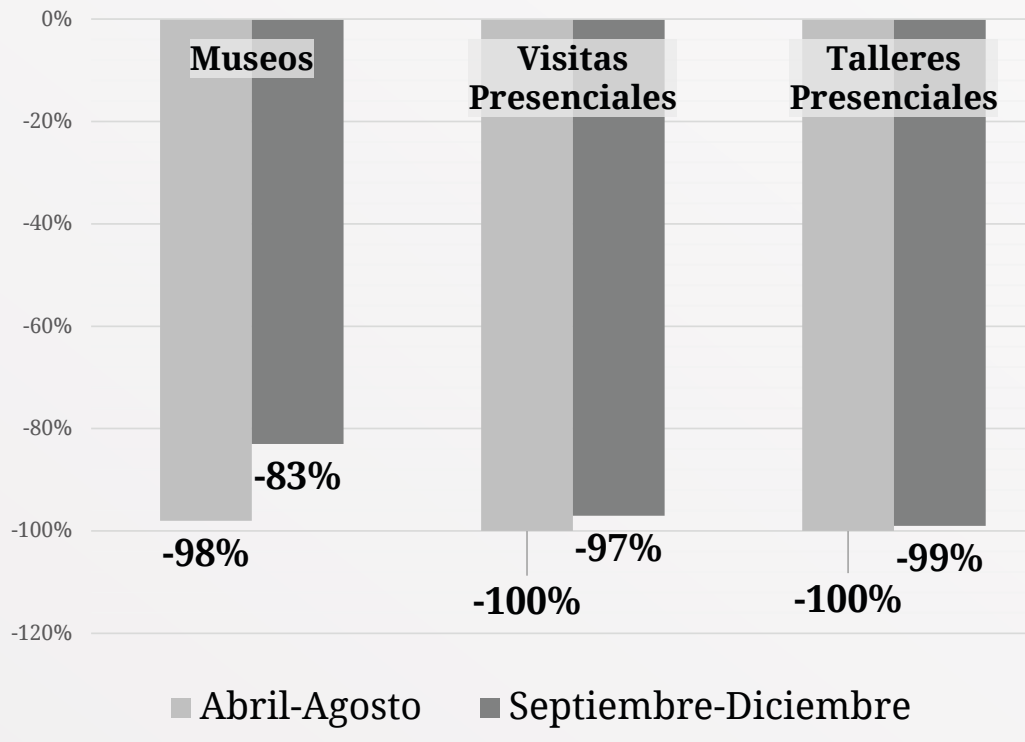

De estas actividades presenciales, principalmente desde los espacios es necesario hacer las diferencias institucionalizados. Durante pertinentes: mientras los la pandemia las iniciativas museos están en un lugar fijo y gubernamentales han sido mínimas, permanente, los talleres o visitas los porcentajes de $3 \%$ en visitas guiadas se preparan ex profeso presenciales y de $1 \%$ en talleres a las convocatorias emitidas, presenciales se debe a propuestas 
individuales y personales de profesores o divulgadores, quienes realizaron grandes esfuerzos, los receptores fueron grupos muy reducidos. Los estados en los cuales se dio esta apertura son Campeche y Chiapas, que pasaron más pronto al semáforo verde ante la pandemia del COVID-19, por lo que se retomaron actividades dentro de sus organismos públicos como el Instituto de Ciencia, Tecnología e Innovación del Estado de Chiapas y en Campeche el Consejo Estatal de Investigación Científica y Desarrollo Tecnológico.

Figura 3.

Temáticas abordadas en la Comunicación de la Ciencia durante la pandemia por COVID-19.

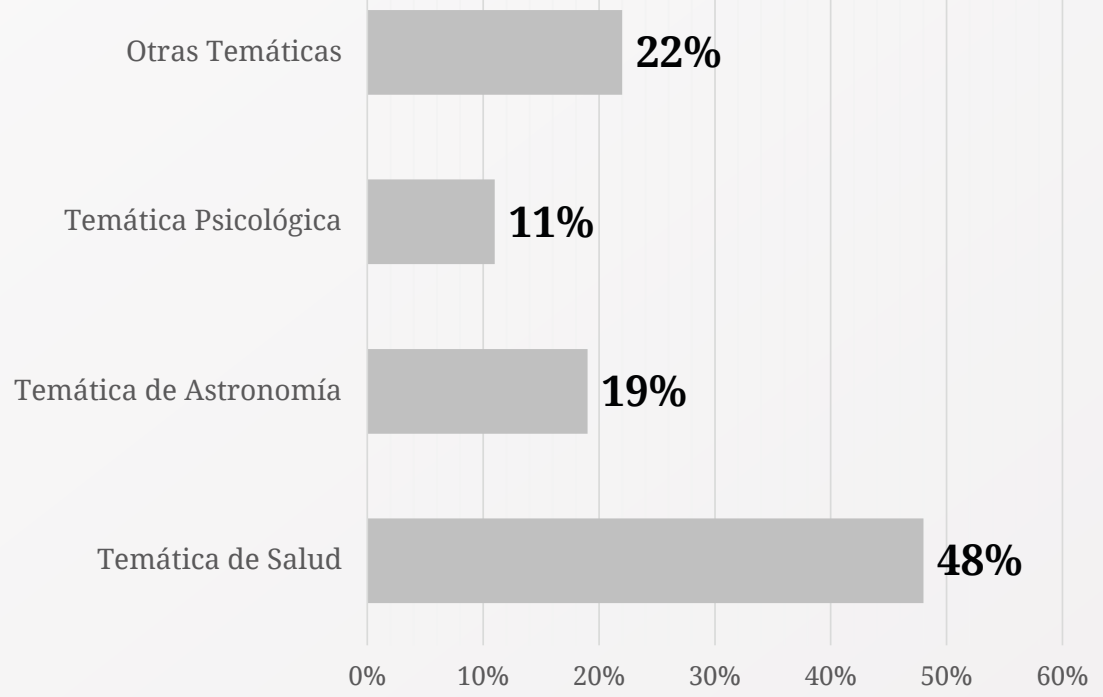

En la figura 3 se presentan los resultados del objeto de estudio que se mencionó en el apartado de la metodología. En la televisión digital abierta, en los periódicos impresos y en los cibermedios se incrementaron los contenidos en torno a la ciencia. En medio de la pandemia los contenidos más desarrollados fueron con la temática de la salud, enfocado en la salud física, ya que la sociedad estaba preocupada de saber en torno a la enfermedades, su protección, los medicamentos, las vacunas, etc. Otro de los temas que integra la agenda de la comunicación de la ciencia fue la astronomía. Esto se presentó en el 2020 por los envíos de cohetes al espacio desde Estados Unidos. 
Un tema que ha recobrado gran relevancia es el de la psicología, enfocado a las emociones; ésto debido a las circunstancias de las y los ciudadanos, ante la cuarentena, ante los cierres temporales de ciertas actividades, ante las consecuencias negativas en lo económico y ante un tema de gran relevancia, como fue la violencia intrafamiliar. En otras temáticas se ubicaron la economía, la física, el derecho, la ingeniería, entre otras.

\section{Figura 4.}

Formas de Comunicación de la Ciencia en la televisión digital abierta, los periódicos impresos y los cibermedios.

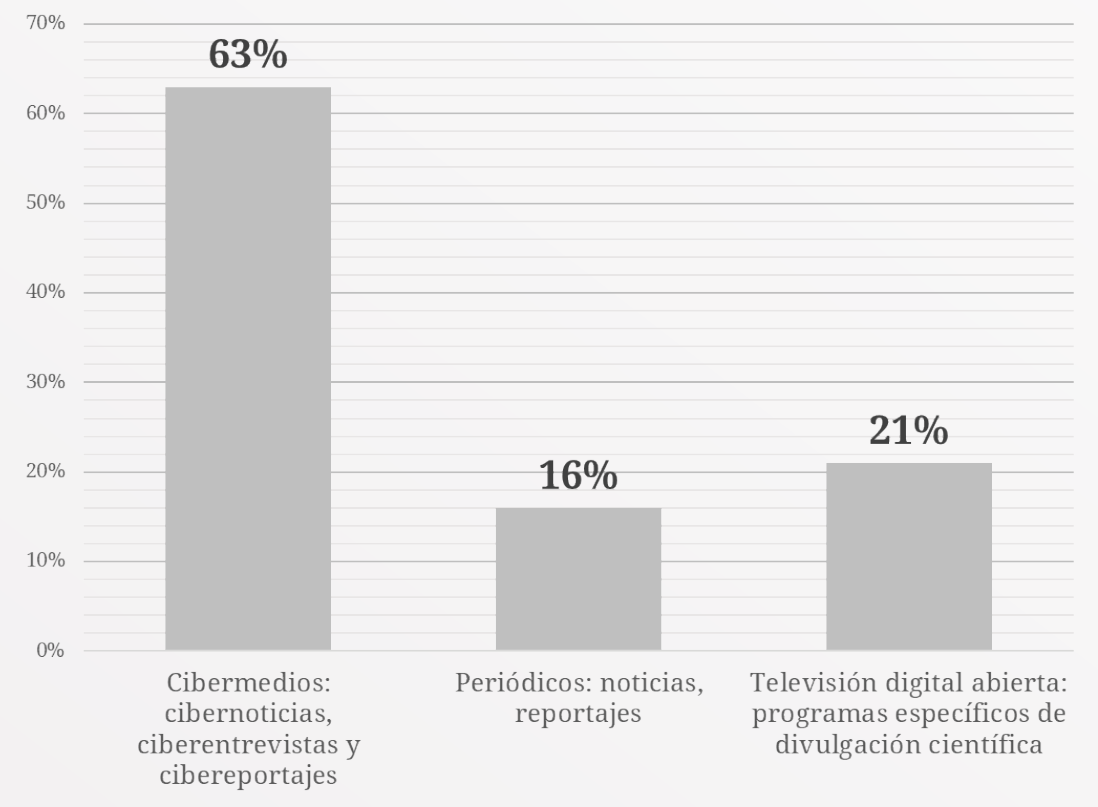

En la televisión digital abierta, impresos fueron las noticias y los en los periódicos impresos y en los cibermedios, los contenidos de comunicación de la ciencia se incrementaron, como lo representa la figura 4. Las cibernoticias, ciberentrevistas y los cibereportajes en los cibermedios, son los géneros periodísticos desarrollados en los espacios digitales. En los periódicos reportajes. En la televisión digital abierta se incrementaron los programas de divulgación científica, en su modalidad de documentales, entrevistas y reportajes.

Aunque el periodismo tradicional no permite la interactividad deseada para la comunicación de la ciencia, 
en los cibermedios sí se tiene dicho elemento, al menos en potencia porque en ocasiones no se realiza. Lo anterior es independiente de la audiencia que se tiene o de la cantidad de usuarios; como se mencionó, en México no se auditan los medios para conocer de manera precisa cuál es su audiencia, situación diferente de lo que sucede en Europa y en Estados Unidos. De hecho, faltan diagnósticos basados en investigación científica, para conocer los resultados de las prácticas de comunicación de la ciencia.

\section{Figura 5.}

Uso de Internet y las redes sociales para la Comunicación de la Ciencia en México.

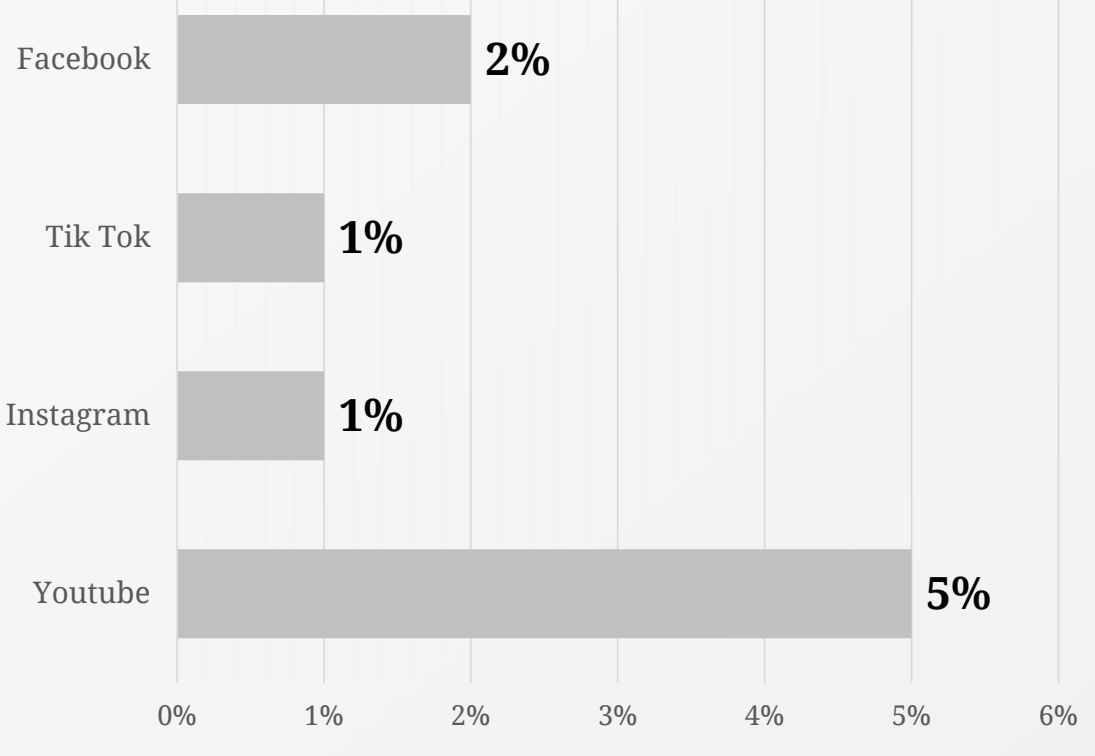

Con base en los resultados de la cierta presencia, como lo representa Asociación de Internet México, las la figura 5.

redes sociales han crecido en el país durante la pandemia (Asociación de Internet MX, 2021). La comunicación de la ciencia ubicada en espacios como Youtube, Redes Sociales como Facebook, Instagram y Tik Tok, tuvo

La divulgación científica desarrollada por divulgadores no ha conseguido en Internet el nivel de desarrollo en esta pandemia, a pesar del incremento en su uso. Además, la comunicación de la 
ciencia aspira a lograr aprendizajes significativos de conocimientos confiables, respaldados por una comunidad científica. Las redes sociales presentan un mínimo de contenidos científicos confiables para su credibilidad; de hecho, son los espacios en los cuales se han ubicado mayor cantidad de "fake news" (noticias falsas). Estamos hablando de los espacios con un mínimo de visitas y publicados por los ciudadanos, que tienen el derecho de hacerlo, pero no la formación.

También aquí prevalecen los aficionados a quienes les gusta hacer contenidos para estos espacios. Entre ellos hay científicos pero los contenidos suelen estar inacabados o, si lo están, tiene carencias respecto a la formación en torno a las competencias comunicativas, las competencias educativas y a las características propias de los espacios digitales. Se consideraron aquellos con más de un millón de visitas en el 2019 y que siguieron publicando en el 2020. No se puede tener el dato exacto en número porque espacios nacen $y$ otros mueren.
En Internet, incluso los espacios de divulgación con mayores visitas en la red carecen todavía de una adaptación al medio (NavarroZamora, 2018). La inmensa mayoría de los sitios se limitan a información en texto, con alguna imagen en el mejor de los casos, y alguno que otro link. Las características que deben incluirse son la convergencia multimedia, la hipertextualidad y una muy importante, la interactividad plena. Además de las características de la comunicación digital, es esencial incorporar los elementos educativos.

Interpretación de la encuesta aplicada a 120 divulgadoras y divulgadores de México

En esta pregunta representada en la figura 6 , se le dieron esas dos únicas opciones, pero no se emitió comentario alguno que añadiera elementos a este cuestionamiento. Antes de la pandemia, la Comunicación de la Ciencia constituía la única fuente de trabajo para el $8 \%$, mientras para el $92 \%$, se integraba como parte del resto de sus actividades profesionales. Esto 
significa que los divulgadores de la ingreso, tuvieron que cambiar ciencia enfocados exclusivamente a otras actividades durante la en esta tarea, al no contar con un pandemia.

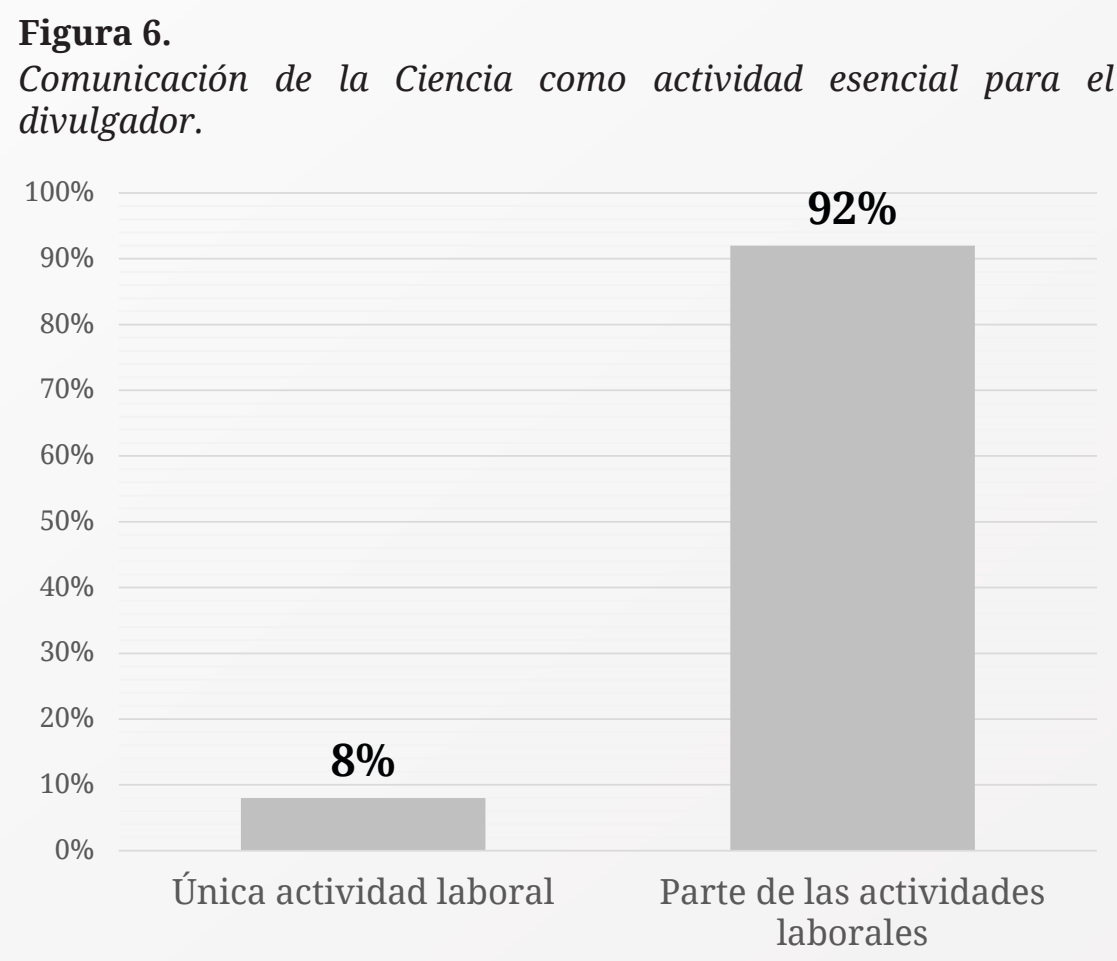

El porcentaje de quienes se dedican en su totalidad a la Comunicación de la Ciencia es muy relevante porque son ellos quienes en su mayoría motivan, organizan y permiten una interacción entre el resto de las y los divulgadores, que desarrollan la actividad parcialmente.

Precisar que la figura 7 es del $92 \%$ de los divulgadores, quienes complementan la divulgación de la ciencia a otra actividad laboral. El mayor porcentaje lo obtuvieron aquellos que laboran en una institución, seguidos por los periodistas y los investigadores en espacios educativos.

Es importante destacar que en el periodismo, solo en la Ciudad de México, Guadalajara y Monterrey, existen periodistas enfocados exclusivamente a cubrir temas de ciencia, integrados en su mayoría en la Red Mexicana de Periodistas de Ciencia (RedMPC), en el resto del país los periodistas cubren otras fuentes, 
generalmente se une la educativa de empresas. A la mayoría de ese y la de salud a la ciencia. También 92\% no afectó la situación de la existe la participación de personas pandemia en su ámbito laboral, ya que trabajan en Organizaciones No que continuaron con el resto de sus Gubernamentales (ONG), así como actividades.

\section{Figura 7.}

¿Dónde trabajan quienes también realizan actividades de Comunicación de la Ciencia?

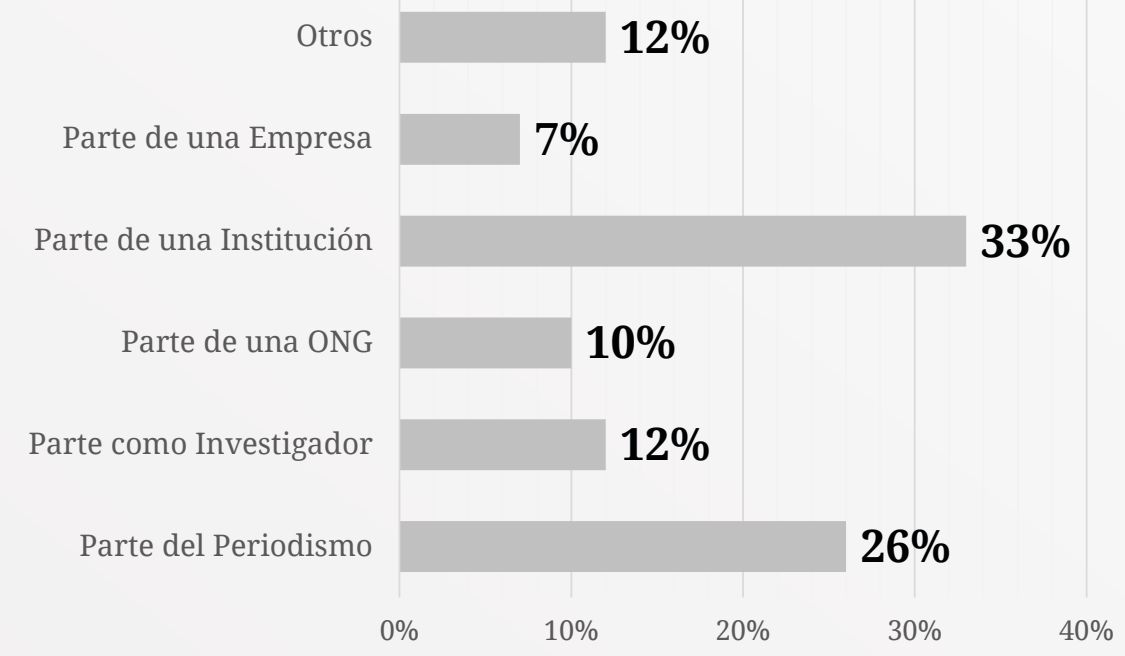

\section{Figura 8.}

Distribución por género de las y los divulgadores encuestados.

Género masculino
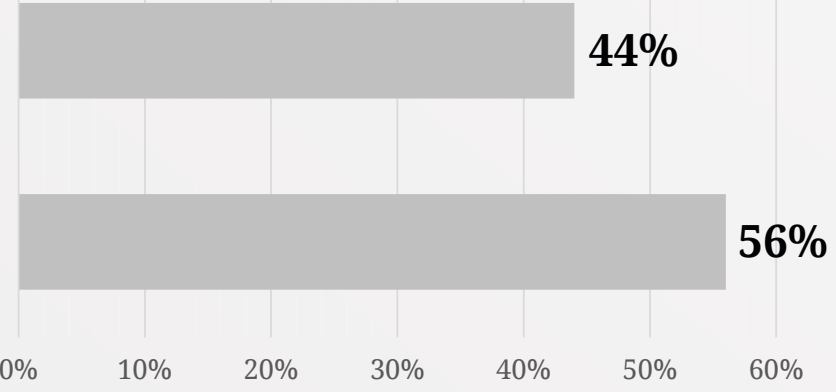
Como puede apreciarse en la figura 8, existe una mayor participación de las mujeres en actividades de divulgación de la ciencia. En este cuestionamiento no se les preguntó el porqué de ello. Es una tendencia que se presenta también en las facultades de comunicación, la presencia del género femenino se ha revertido $\mathrm{y}$ es mayor; una de las ventajas de ello es el enfoque de género que se puede dar en esta actividad, para su mayor desarrollo. Tanto en hombres como en mujeres ubicamos competencias para el desarrollo de la divulgación de la ciencia.

\section{Figura 9.}

Edades de los divulgadores de la Ciencia.

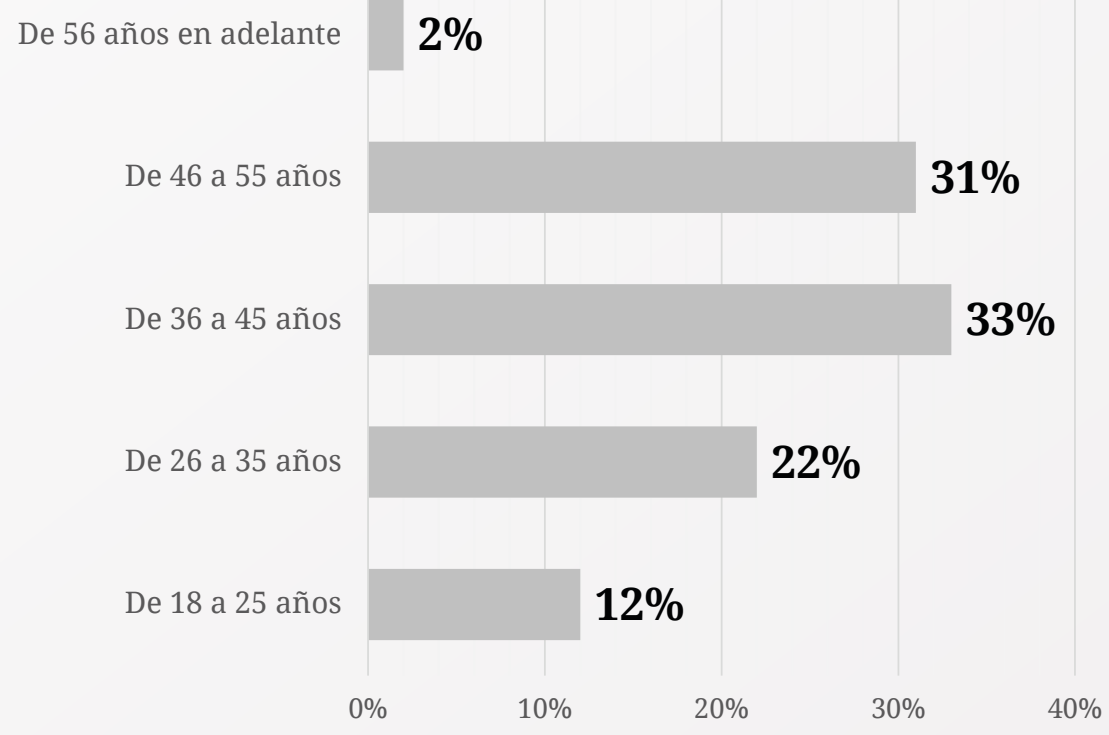

Esta figura 9 refleja las edades de La formación, representada en las y los divulgadores de la Ciencia, la figura10, permite ubicar un aquí se ve una presencia mayor de elemento importante para la los compañeros de los 36 a los 45 años divulgación de la Ciencia, tener y de los 46 a los 55 años. Una de las inferencias es que a dichas edades ya se presenta una consolidación en el ámbito laboral, sea en espacios públicos o en los privados. una formación de Licenciatura da elementos claves, el posgrado da mayores fortalezas como investigador y no como divulgador, sin embargo aquí no se incluyen 
las competencias de comunicación Comunicación de la Ciencia, además y educación, pilares de la del conocimiento científico.

Figura 10.

Formación Académica de los divulgadores de la ciencia.

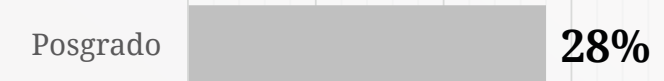

Licenciatura

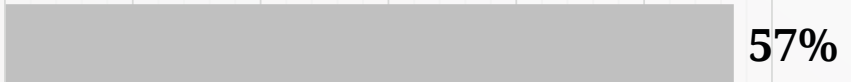

No Licenciatura

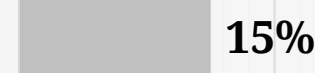

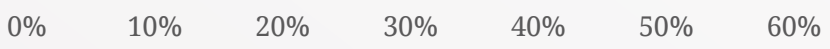

En la figura 11 se representa que el un elemento significativo ya que son 89\% de divulgadores de la ciencia fundamentales para la transferencia encuestados no tienen formación en del conocimiento y el logro de un comunicación y educación. Éste es aprendizaje.

\section{Figura 11.}

Formación de los Divulgadores de la Ciencia en educación y comunicación.

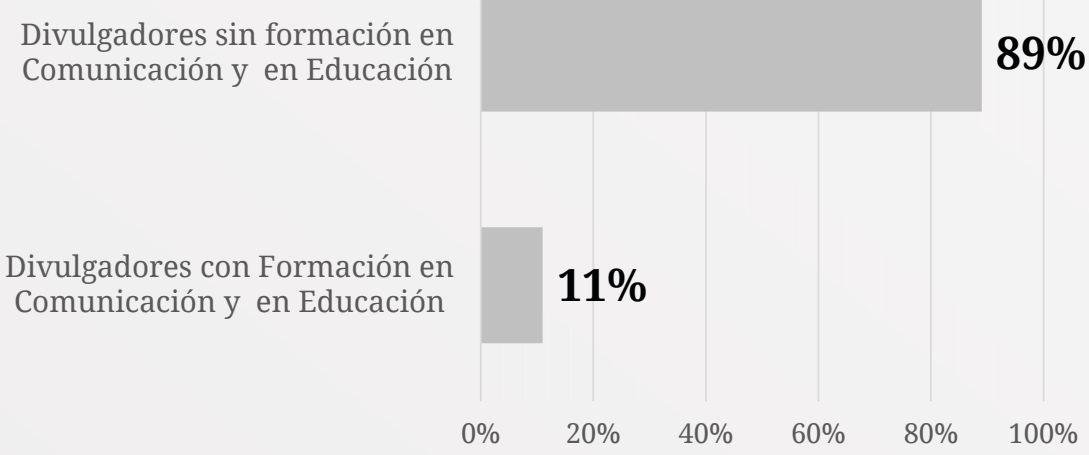


Dentro del perfil del divulgador se deben tener aptitudes, que son tanto las capacidades y las actitudes, como la disposición necesaria para la Comunicación de la Ciencia. Por otra parte, la mayoría de los docentes y los investigadores de educación media superior y superior que realizan divulgación, tiene una nula formación en comunicación y en educación. Para la divulgación de la ciencia es importante tener una licenciatura o un posgrado por el conocimiento disciplinar, pero no se hace una Comunicación de la Ciencia asertiva si no se tienen otras competencias en la educación y en la comunicación.

Figura 12.

¿Recibieron algún tipo de capacitación durante la pandemia del COVID-19?

No recibieron capacitación

$97 \%$

Sí recibieron capacitación

$3 \%$

$\begin{array}{llllll}0 \% & 20 \% & 40 \% & 60 \% & 80 \% & 100 \%\end{array}$

Las y los divulgadores de la Ciencia deben tener una actualización constante y pertinente en los dos grandes ámbitos de la educación y la comunicación. En la figura 12 se refleja que durante esta pandemia tampoco hubo una formación y actualización de los divulgadores de la Ciencia, sólo el 3\% recibió cursos de capacitación.
Del 97\%, la mayoría comentó que no hubo oferta de parte de las instituciones públicas para formarlos. Esto también refleja que no hay una formación para hacer frente a la realidad actual de la pandemia en torno a la Comunicación de la Ciencia, se requiere que los divulgadores de la Ciencia cuenten con una capacitación, para estar preparados para el retorno. 
La comunicación de la ciencia en la pandemia por COVID-19 y sus divulgadores

Lizy Navarro Zamora

\section{Figura 13.}

Evaluación de las actividades mediadas por las TIC con éxito o sin éxito.

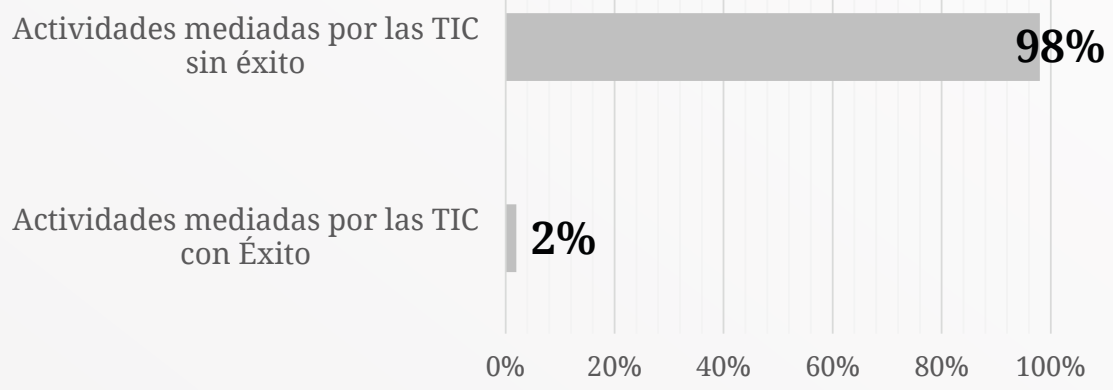

De las personas encuestadas que realizaron actividades mediadas por las TIC, que fueron un número muy reducido, alrededor del $2 \%$ consideraron que tuvieron éxito en ellas y el $98 \%$ que no tuvieron éxito, manifestaron el muy bajo acceso del público a éstas, como se representa en la figura 13 . Una de las razones que argumentaron fue la saturación de oferta, en el caso de los estudiantes por recibir sus lecciones en televisión o en computadora.
Los que manifestaron éxito en las actividades mediadas por las TIC expresaron que antes habían tenido comunicación personal con los que participaron. La interactividad y la construcción, ex profeso para estos espacios, es casi nula. Además, el exceso de conferencias, pláticas, entrevistas, mediadas por las TIC se convirtió en un desorden comunicativo y educativo. Las plataformas no permiten integrar una agenda de actividades de Comunicación de la Ciencia.

Figura 14.

¿Cuál es el público que consideras se ve más afectado por la reducción de las actividades de Comunicación de la Ciencia?

Otros

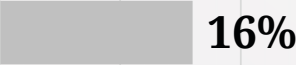

Público Adolescente y Juvenil

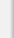
$44 \%$

Público Infantil

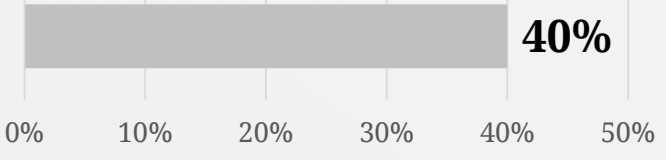


Con base en la experiencia de los 120 divulgadores de la Ciencia encuestados, representados en la figura 14, el público más afectado por la reducción de la comunicación de la Ciencia presencial es el adolescente, juvenil e infantil. No hay estadística del consumo de la Comunicación de la Ciencia en la televisión abierta digital, en los medios impresos y en los cibermedios, por ello es a partir de los divulgadores que se pueden obtener algunas conclusiones como esta. De las actividades presenciales como museos, talleres y visitas, quienes más asistían en el año 2019 eran las y los niños; las y los adolescentes, en el 2020 fueron mínimas y como ya mencionamos se destacan estados como Chiapas y Campeche que fueron las primeras entidades en pasar a semáforo verde, el nivel más bajo de contagios y hospitalizaciones por la COVID-19.

Figura 15.

Actividades de Comunicación de la Ciencia planeadas para el regreso a la nueva normalidad.

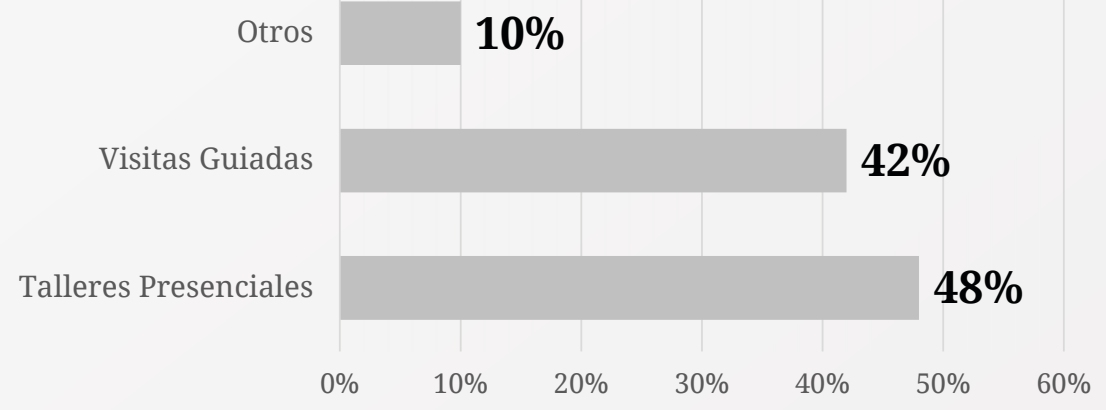

Esto refleja la tradición de la contacto directo, privilegiando la Comunicación de la Ciencia por comunicación. los divulgadores que es a través de los talleres presenciales y las visitas guiadas. La interpretación que se da a ello es la tradición que se tiene en las actividades de la divulgación de la ciencia; estas Con estas respuestas también se recomienda la formación para la Comunicación de la Ciencia en espacios digitales, sobre todo por actividades permitirán además un el perfil de las y los niños, de las y los adolescentes, es necesario 
incrementar la oferta lúdica, basada en la pedagogía y comunicación digital.

\section{|Conclusiones}

La sociedad nacional y global debe estar construida a partir de los conocimientos científicos. Con desafíos como la pandemia del COVID-19, se requieren expertos en ciencia y en tecnología altamente formados para responder a la demanda social, así como expertos en divulgación de la ciencia para transmitir estos conocimientos.

La presente investigación refleja diversas realidades en México en torno a la Comunicación de la Ciencia durante la pandemia COVID-19, de marzo a diciembre del 2020. Algunas conclusiones son:

- La Comunicación de la Ciencia prácticamente fue mínima ante la necesidad social y se desarrolló en los medios masivos de comunicación como la televisión digital abierta, los periódicos impresos y los cibermedios.

- En los medios masivos de comunicación como la televisión digital abierta, los periódicos impresos y los cibermedios, se realizaron actividades de periodismo científico, tales como noticias, entrevistas, crónicas, reportajes, columnas, artículos y editoriales; con su paralelo en los cibermedios.

- A partir de la investigación representada en las figuras; en México, el desarrollo de la Comunicación de la Ciencia en las redes sociales fue mínima y con un contenido sin producción comunicativa, ni elementos educativos. Si se consideran espacios de visitas significativas, en las redes sociales hablamos de más de un millón de visitas. No son las publicaciones de grupos pequeños; la Comunicación de la Ciencia no sólo debe ser abierta, debe ser visitada. Miles y millones de personas escribieron sobre la COVID-19, pero eso no es Comunicación de la Ciencia.

- Es necesaria una formación y actualización del divulgador de la Ciencia ante la Pandemia del COVID-19. 
- Con un mínimo de actividades de divulgación de la ciencia realizadas, se infiere que la niñez y la juventud no recibieron los aprendizajes significativos que cada año tenían, lo cual se convierte en retrocesos y desafíos-metas una vez que termine la pandemia. La apropiación de la ciencia es y será esencial.

- Para la población con nulo acceso a Internet, se sugiere emplear la televisión sin costo como se hizo con la educación institucionalizada para incorporar más Comunicación de la Ciencia, sobre todo en los canales analizados de Televisa y de Televisión Azteca, así se podrá incrementar la penetración.

- El desarrollo de la comunicación de la ciencia pudo haber jugado un rol fundamental para comprender y entender el fenómeno de la pandemia COVID-19; sin embargo las precauciones para evitar los contagios anularon las actividades presenciales $\mathrm{y}$, en las mediadas por las TIC, los divulgadores no estaban formados para la construcción de contenidos para dichos espacios. Una vez que se restablezca la normalidad, se sugiere que en el 2021 se les forme $\mathrm{y}$ actualice a los divulgadores en comunicación, educación y uso de las TIC.

- La Comunicación de la Ciencia debe darse en los espacios que tengan mayor audiencia, tanto en los medios masivos de comunicación, los mediados por Internet y de hecho los presenciales. Si un museo abrió su plataforma en Internet pero no tuvo visitas contables $\mathrm{y}$ significativas, no tiene relevancia. 
La comunicación de la ciencia en la pandemia por COVID-19 y sus divulgadores

Lizy Navarro Zamora

\section{| Referencias}

Asociación de Internet MX. (2021, 27 de enero). Estudio de los Hábitos de los usuarios de Internet en México AIMX 2020. https://www. asociaciondeinternet.mx/estudios/asociacion

Ataide-Malcher, A. \& Cunha-López, S. (2013). Construyendo una noción de comunicación de la ciencia. Chasqui, 122, (74-81). https://cutt.ly/pnkVPhB

Calvo-Hernando, M. (2003). Divulgación y Periodismo Científico: entre la claridad y la exactitud. Universidad Nacional Autónoma de México.

Corredor-Trejo, N. \& Socorro, M. (2014). Impacto de las tecnologías de información y comunicación en la divulgación del conocimiento científico. Cieg, (5)2, 139-161.

Martínez-Albertos, J. L. (1997). El Ocaso del Periodismo. CIMS.

Martínez-Mendoza, S. (2012). La Difusión y la Divulgación de la Ciencia en Chiapas. Razón y Palabra, 78. https://cutt.ly/pnrl0tQ

Moreira, M. A. (2012). ¿Al final, qué es el aprendizaje significativo? Qurriculum, 25, 29-56. https://cutt.ly/gnrlBoO

Navarro-Zamora, L. (2002). Los periódicos On line. Universidad Autónoma de San Luis Potosí.

Navarro-Zamora, L. (2018). Elementos del Ciberperiodismo en Iberoamérica. Sociedad Latina de Comunicación Social.

Pacheco-Muñoz, M. F. (2003). La divulgación de la ciencia. Ciencias, 71, 56-64.

Organización de las Naciones Unidas para la Educación, la Ciencia y la Cultura [UNESCO] (2020). Museos. https://cutt.ly/xnkMGMg

Organización de las Naciones Unidas para la Educación, la Ciencia y la Cultura [UNESCO]. (2018). Construir Sociedades del Conocimiento. https://cutt.ly/6nyoz80

Vygotsky, L. (1984). Aprendizaje y desarrollo intelectual en la edad escolar. Escritos escogidos de Psicología, 27/28, 105-116. https://dialnet.unirioja.es/servlet/articulo?codigo $=668448$ 